\title{
Tumor heterogeneity: a central foe in the war on cancer
}

\author{
Jane de Lartigue, $\mathrm{PhD}$
}

\begin{abstract}
A major challenge to effective cancer treatment is the astounding level of heterogeneity that tumors display on many different fronts. Here, we discuss how a deeper appreciation of this heterogeneity and its impact is driving research efforts to better understand and tackle it and a radical rethink of treatment paradigms.
\end{abstract}

\section{A complex and dynamic disease}

The nonuniformity of cancer has long been appreciated, reflected most visibly in the variation of response to the same treatment across patients with the same type of tumor (inter-tumor heterogeneity). The extent of tumor heterogeneity is being fully realized only now, with the advent of next-generation sequencing technologies. Even within the same tumor, there can be significant heterogeneity from cell to cell (intra-tumor heterogeneity), yielding substantial complexity in cancer.

Heterogeneity reveals itself on many different levels. Histologically speaking, tumors are composed of a nonhomogenous mass of cells that vary in type and number. In terms of their molecular make-up, there is substantial variation in the types of molecular alterations observed, all the way down to the single cell level. In even more abstract terms, beyond the cancer itself, the microenvironment in which it resides can be highly heterogeneous, composed of a plethora of different supportive and tumor-infiltrating normal cells.

Heterogeneity can manifest spatially, reflecting differences in the composition of the primary tumor and tumors at secondary sites or across regions of the same tumor mass and temporally, at different time points across a tumor's natural history. Evocative of the second law of thermodynamics, cancers generally become more diverse and complex over time. ${ }^{1-3}$

\section{A tale of 2 models}

It is widely accepted that the transformation of a normal cell into a malignant one occurs with the acquisition of certain "hallmark" abilities, but there are myriad ways in which these can be attained. Two key models can be used to explain how tumors develop - the clonal evolution model and the cancer stem cell (CSC) model (Figure 1).

\section{The clonal evolution model}

As cells divide, they randomly acquire mutations as a result of DNA damage. The clonal evolution model posits that cancer develops as the result of a multistep accumulation of a series of "driver" mutations that confer a promalignant advantage to the cell and ultimately fuel a cancerous hallmark.

This evolution can occur in a linear fashion, whereby the emergence of a new driver mutation conveys such a potent evolutionary advantage that it outcompetes all previous clones. There is limited evidence for linear evolution in most advanced human cancers; instead, they are thought to evolve predominantly through a process of branching evolution, in which multiple clones can diverge in parallel from a common ancestor through the acquisition of different driver mutations. This results in common clonal mutations that form the trunk of the cancer's evolutionary tree and are shared by all cells and subclonal mutations, which make up the branches and differ from cell to cell.

More recently, several other mechanisms of clonal evolution have been proposed, including neutral evolution, a type of branching evolution in which there are no selective pressures and evolution occurs by random mutations occurring over time that lead to genetic drift, and punctuated evolution, in which there are short evolutionary bursts of hypermutation. ${ }^{4,5}$

\section{The CSC model}

This model posits that the ability to form and sustain a cancer is restricted to a single cell type - the cancer stem cells - which have the unique capacity for selfrenewal and differentiation. Although the forces of evolution are still involved in this model, they act on a hierarchy of cells, with stem cells sitting at the top. A tumor is derived from a single stem cell that has acquired a mutation, and the heterogeneity observed results both from the differentiation and the accumulation of mutations in CSCs. 


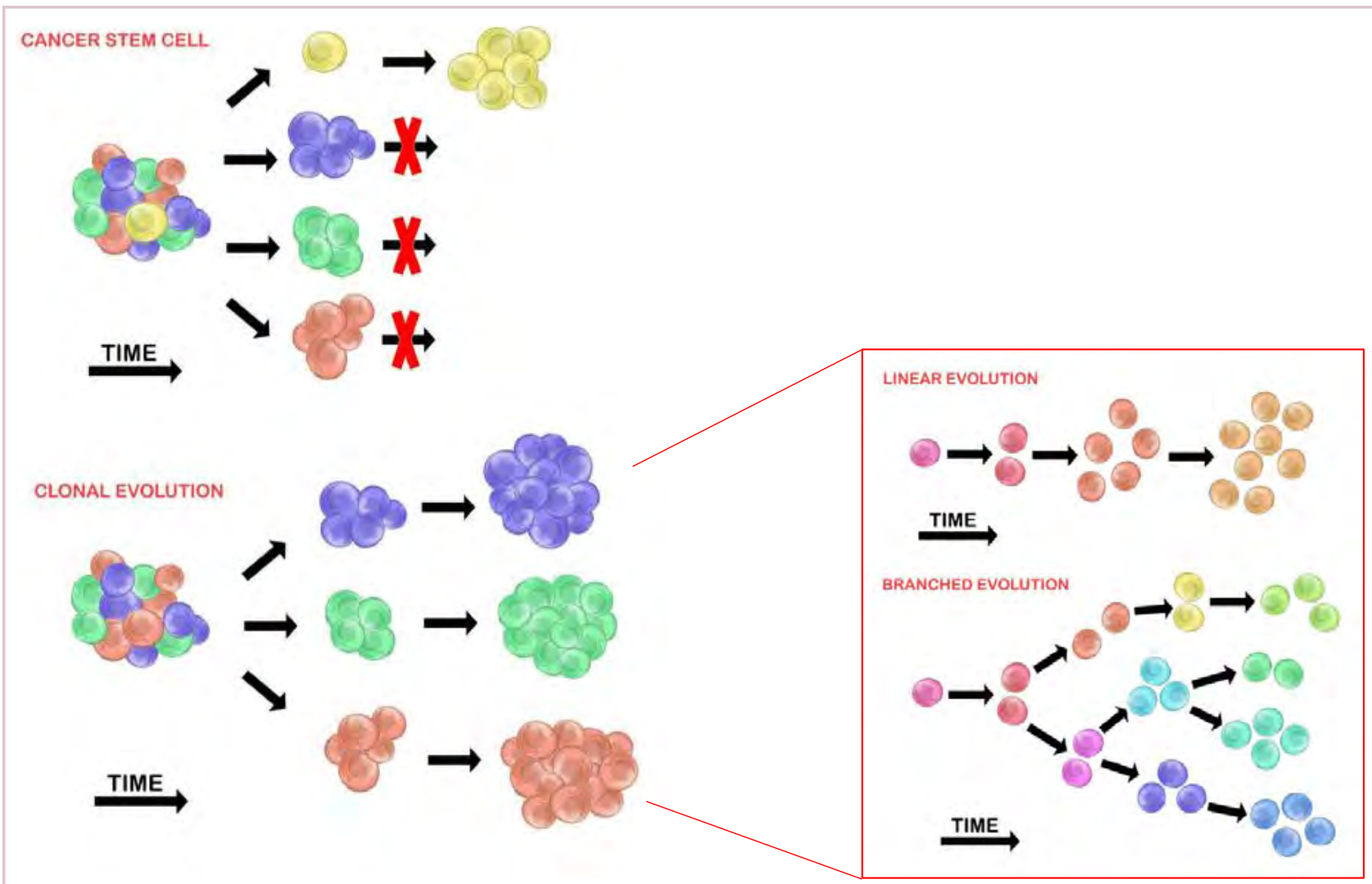

FIGURE 1 Evolution of heterogeneity over time. There are 2 prevailing models of tumor development that have implications for how heterogeneity evolves over time: the cancer stem cell model and the clonal evolution model, which are not mutually exclusive. The former posits that only a select few cancer cells, the cancer stem cells, have the potential to form new tumor cells and it is variability within these cells that gives rise to the heterogeneity observed in the tumors to which they give rise. In the clonal evolution model, tumor cells arise from a single mutated cell and acquire additional varied mutations as they progress. This can occur in a linear fashion, whereby the cells successively acquire mutations that confer a growth or survival advantage, or through a branched mechanism, giving rise to multiple genetically diverse subclonal populations. Available at https://en.wikipedia.org/wiki/Tumour_heterogeneity. Last update February 27, 2014. Accessed May 1, 2018. Reproduced under a Creative Commons Attribution ShareAlike License.

Accumulated experimental evidence suggests that these models are not mutually exclusive and that they can all contribute to heterogeneity in varied amounts across different tumor types. What is clear is that heterogeneity and evolution are intricately intertwined in cancer development. . $^{1,2,6}$

\section{An unstable genome}

Heterogeneity and evolution are fueled by genomic alterations and the genome instability that they foster. This genome instability can range from single base pair substitutions to a doubling of the entire genome and results from both exposure to exogenous mutagens (eg, chemicals and ultraviolet radiation) and genomic alterations that have an impact on important cellular processes (eg, DNA repair or replication).

Among the most common causes of genome instability are mutations in the DNA mismatch repair pathway proteins or in the proofreading polymerase enzymes. Genome instability is often associated with unique mutational signa- tures - characteristic combinations of mutations that arose as the result of the specific biological processes underlying them. ${ }^{7}$

Genome-wide analyses have begun to reveal these mutational signatures across the spectrum of human cancers. The Wellcome Sanger Institute's Catalogue of Somatic Mutations in Cancer (COSMIC) database has generated a set of 30 mutational signatures based on analysis of almost 11,000 exomes and more than 1,000 whole genomes spanning 40 different cancer types, some of which have been linked with specific mutagenic processes, such as tobacco, UV radiation, and DNA repair deficiency (Table 1). ${ }^{8}$

One potential downside to genome instability for cancer cells is that it can lead to massive deleterious effects that overwhelm the genome and lead to cell death. A potential way to overcome this is for the changes to be restricted to a small portion of the genome and there is evidence for this in the discovery of patterns of localized hypermutation (kataegis) described in breast cancer genomes and in several 
TABLE 1 COSMIC mutational signatures in cancer $^{a}$

\section{Process}

Associated signatures

Age-related mutagenesis

Signature 1

All cancer types, most samples

Signature 5

All cancer types, most samples

Homologous recombination deficiency

\author{
Signature 3 \\ Breast, ovarian, and pancreatic \\ cancers
}

\section{Description}

- Associated with small numbers of small insertions and deletions, resulting from spontaneous deamination of 5-methylcytosine

- Associated with a predominance of $\mathrm{T}>\mathrm{C}$ substitutions in the ApTpN trinucleotide context with transcriptional strand bias, thought to result from loss of the FHIT gene

Associated with an increased number of large insertions and deletions with microhomology at the breakpoints. Related to a failure of DNA double-strand break repair by homologous recombination leg, $B R C A 1 / 2$ mutations)

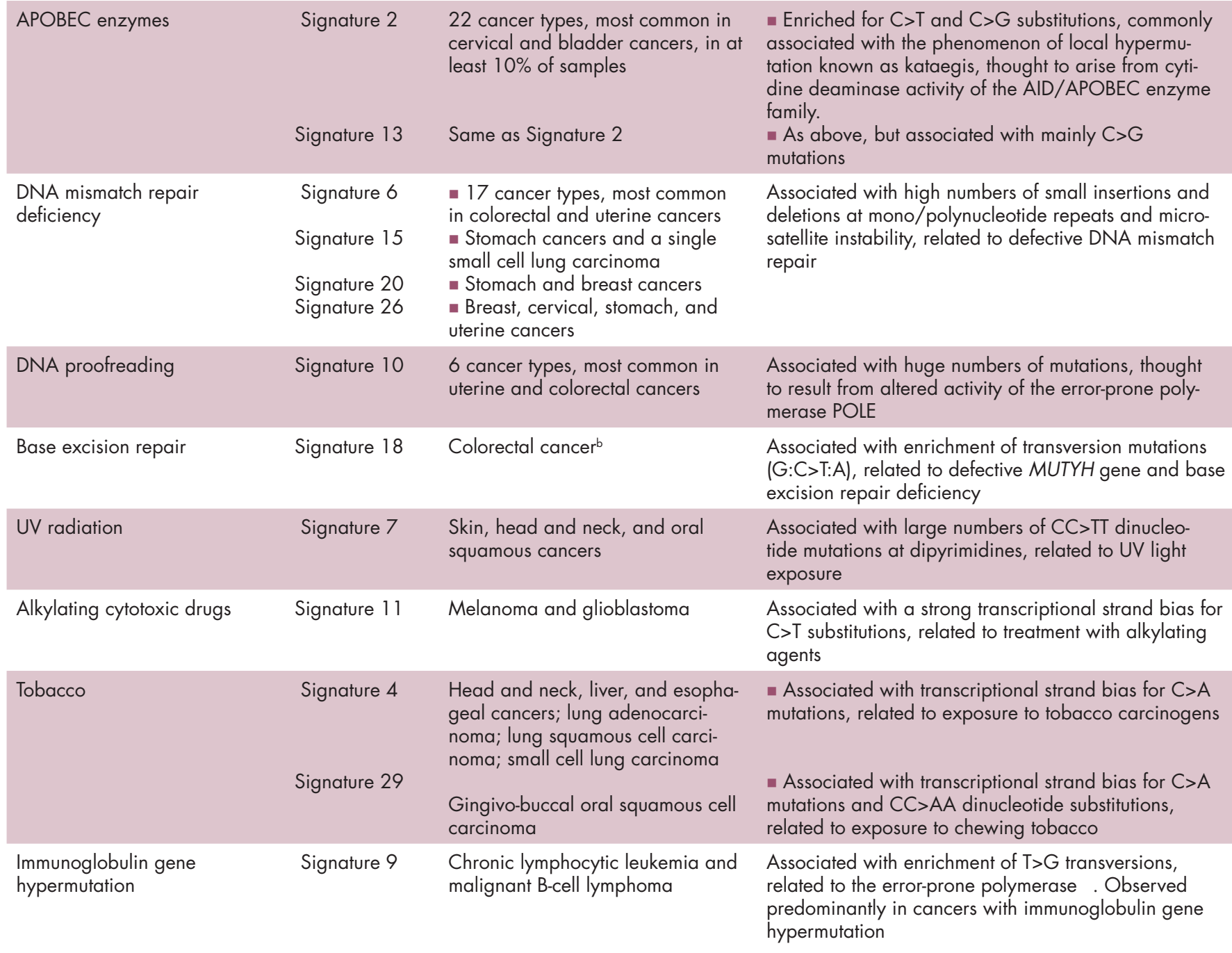

aReferences: Wellcome Sanger Institute. Signatures of mutational processes in human cancer. https://cancer.sanger.ac.uk/cosmic/signatures. Update/publication date not available. Accessed May 1, 2018. Volinia S, et al. The ubiquitous 'cancer mutational signature' 5 occurs specifically in cancers with deleted FHIT alleles. Oncotarget. 2017;8(60):102199-102211. Pilati C, et al. Mutational signature analysis identifies MUTYH deficiency in colorectal cancers and adrenocortical carcinomas. J Pathol. 2017;242:10-15. bSignature 18 has been observed in other cancer types, but it has not yet been linked to base excision repair in those cases. 


\section{Feature}

TABLE 2 Targeting DNA repair pathways

\section{Drug}

Olaparib

(Lynparza)

Manufacturer

AstraZeneca
PARP inhibitor

Mechanism of action

\section{Most advanced clinical setting (clinicaltrials.gov identifier)}

FDA approved

BRCA-mutated ovarian cancer (2014)

Maintenance therapy ovarian cancer (2017)

BRCA-mutated metastatic breast cancer (2018)

Phase 3

BRCA-mutated pancreatic cancer (POLO; NCT02184195)

$\begin{array}{lll}\text { Niraparib } & \text { Tesaro } & \text { PARP inhibitor } \\ \text { (Zejula) } & & \end{array}$

FDA approved

Maintenance therapy ovarian cancer

Phase 3

Maintenance therapy SCLC (NCT03516084)

Maintenance therapy ovarian cancer (NCT02655016)

\begin{tabular}{|c|c|c|c|}
\hline $\begin{array}{l}\text { Rucaparib } \\
\text { (Rubraca) }\end{array}$ & Pfizer & PARP inhibitor & $\begin{array}{l}\text { FDA approved } \\
\text { BRCA-mutated ovarian cancer (2016) } \\
\text { Maintenance therapy ovarian cancer (2018) } \\
\text { Phase } 3 \\
\text { BRCA-mutated ovarian cancer (ARIEL-4; NCTO2855944) } \\
\text { HRD-positive mCRPC (TRITON3; NCTO2975934) }\end{array}$ \\
\hline Veliparib & Abbott & PARP inhibitor & $\begin{array}{l}\text { Phase } 3 \\
\text { In combination with temozolomide in GBM (NCT02152982) }\end{array}$ \\
\hline Talazoparib & BioMarin & PARP inhibitor & $\begin{array}{l}\text { Phase } 2 \\
\text { BRCA/PTEN/HRD-positive cancers (NCT02286687) } \\
\text { BRCA-wildtype TNBC/solid tumors (NCTO2401347) } \\
\text { HRD-positive squamous cell lung cancer (NCT03377556) } \\
\text { mCRPC (NCT03148795) }\end{array}$ \\
\hline MSC2490484A & EMD-Serono & DNA-PK inhibitor & $\begin{array}{l}\text { Phase } 1 \\
\text { In combination with RT in advanced cancers (NCT02516813) }\end{array}$ \\
\hline CC-115 & Celgene & DNA-PK inhibitor & $\begin{array}{l}\text { Phase } 1 \\
\text { In combination with enzalutamide in mCRPC (NCT02833883) }\end{array}$ \\
\hline AZD0156 & AstraZeneca & ATM inhibitor & $\begin{array}{l}\text { Phase } 1 \\
\text { In advanced cancer (AToM; NCT02588105) }\end{array}$ \\
\hline VX-970 & Vertex & ATR inhibitor & $\begin{array}{l}\text { Phase } 2 \\
\text { Urothelial cancer (NCT02567409) } \\
\text { Ovarian cancer (NCT02627443) }\end{array}$ \\
\hline AZD6738 & AstraZeneca & ATR inhibitor & $\begin{array}{l}\text { Phase } 2 \\
\text { In combination with olaparib in SCLC (SUKSES-N2; NCT03428607) } \\
\text { In combination with acalabrutinib in CLL (NCTO3328273) }\end{array}$ \\
\hline $\begin{array}{l}\text { Prexasertib } \\
\text { (LY2606368) }\end{array}$ & Eli Lilly & CHK $1 / 2$ inhibitor & $\begin{array}{l}\text { Phase } 2 \\
\text { SCLC (NCT02735980) } \\
\text { BRCA-mutated breast or ovarian cancer or CRPC (NCTO2203513) } \\
\text { Solid tumors with replicative stress or HRD (NCTO2873975) } \\
\text { Ovarian cancer (NCTO3414047) }\end{array}$ \\
\hline AZD 1775 & AstraZeneca & Weel kinase inhibitor & $\begin{array}{l}\text { Phase } 2 \\
\text { SCLC (NCT02688907) } \\
\text { In combination with cisplatin in breast cancer (NCT03012477) } \\
\text { +/- cytarabine in AML or MDS (NCT02666950) }\end{array}$ \\
\hline $\begin{array}{l}\text { Nivolumab } \\
\text { (Opdivo) }\end{array}$ & $\begin{array}{l}\text { Bristol-Myers } \\
\text { Squibb }\end{array}$ & Immune checkpoint inhibitor & $\begin{array}{l}\text { FDA approved } \\
\text { MSI-H or dMMR CRC (2017) } \\
\text { Phase } 2 \\
\text { Prostate cancer with DNA repair defects } \\
\text { (ImmunoProst; NCTO3040791) } \\
\text { Uterine cancer with dMMR/MSI-H (NCT03241745) }\end{array}$ \\
\hline $\begin{array}{l}\text { Pembrolizumab } \\
\text { (Keytruda) }\end{array}$ & Merck & Immune checkpoint inhibitor & $\begin{array}{l}\text { FDA approved } \\
\text { MSI-H or dMMR cancers (2017) } \\
\text { Phase } 2 \\
\text { mCRPC with DNA damage repair defects (NCT03248570) }\end{array}$ \\
\hline
\end{tabular}

Continued from on following page 
Table 2 continued from previous page

\begin{tabular}{|c|c|c|c|}
\hline Drug & Manufacturer & Mechanism of action & Most advanced clinical setting (clinicaltrials.gov identifier \\
\hline $\begin{array}{l}\text { Atezolizumab } \\
\text { (Tecentriq) }\end{array}$ & Genentech & Immune checkpoint inhibitor & $\begin{array}{l}\text { Phase } 3 \\
\text { dMMR CRC in combination with bevacizumab and chemotherapy } \\
\text { (NCT02997228) }\end{array}$ \\
\hline $\begin{array}{l}\text { Durvalumab } \\
\text { (Imfinzi) }\end{array}$ & AstraZeneca & Immune checkpoint inhibitor & $\begin{array}{l}\text { Phase } 2 \\
\text { MSI-H or POLE-mutated mCRC (NCT03435107) }\end{array}$ \\
\hline
\end{tabular}

AML, acute myeloid leukemia; ATM, ataxia telangiectasia mutated protein; ATR, ataxia telangiectasia and rad3 related protein; BRCA, breast cancer susceptibility gene; CHK1/2, checkpoint kinase 1/2; CLL, chronic lymphocytic leukemia; CRC, colorectal cancer; dMMR, defective mismatch repair; GBM, glioblastoma; HRD, homologous recombination deficiency; MDS, myelodysplastic syndrome; MSI-H, microsatellite instability-high; PARP, poly(ADP)ribose polymerase; POLE, DNA polymerase epsilon; PTEN, phosphatase and tensin homolog; RT, radiation therapy; SCLC, small cell lung cancer

novel classes of chromosomal rearrangements described in other genome sequencing studies (eg, chromothripsis and chromoplexy). ${ }^{9}$

\section{Fueling resistance}

Arguably, heterogeneity presents one of the most significant barriers to effective cancer therapy, and this has become increasingly true in the era of personalized medicine in which targeted therapies take aim at specific molecular abnormalities.

It is vital that drugs target the truncal alterations that are present in all cancer cells to ensure that the entire cancer is eradicated. However, it is not always possible to target these alterations, for example, at the present time tumor suppressor proteins like p53 are not druggable.

Even when truncal alterations have been targeted successfully, such as epidermal growth factor receptor $(E G F R)$ mutations and anaplastic lymphoma kinase $(A L K)$ chromosomal rearrangements in non-small-cell lung cancer (NSCLC) and $B R A F$ mutations in melanoma, the longterm efficacy of these drugs is almost invariably limited by the development of resistance.

Tumor heterogeneity and the clonal evolution it fuels are central drivers of resistance. Because tumors are dynamic and continue to evolve, anticancer treatments can act as a strong selective pressure and drive the emergence of drugresistant subclones that allow the tumor to persist. In fact, study findings have revealed that small populations of resistant cells may be present before treatment. Thus, resistance may also occur as a result of the outgrowth of preexisting treatment-resistant cells that suddenly find that they acquire a survival advantage in the presence of a drug. ${ }^{1,6}$

\section{Tackling heterogeneity}

Despite extensive clinical documentation of the existence of heterogeneity and its underlying mechanisms across a range of tumor types, the development of novel clinical trial designs and therapeutic strategies that account for its effects have only recently begun to be explored.

For the most part, this was because of a lack of effective methods for evaluating intratumor heterogeneity.
Multiregion biopsies, in which tissue derived from multiple different regions of a single tumor mass or from distinct cancerous lesions within the same patient, give a snapshot of tumor heterogeneity at a single point in time. The repeated longitudinal sampling required to gain a deeper appreciation of tumor heterogeneity over the course of tumor evolution is often not possible because of the morbidity associated with repeated surgical procedures.

Liquid biopsies, in which DNA sequencing can be performed on tumor components that are found circulating in the blood of cancer patients (including circulating tumor cells and cell-free circulating tumor DNA) have rapidly gained traction in the past several decades and offer an unprecedented opportunity for real-time assessment of evolving tumor heterogeneity.

They have proved to be highly sensitive and specific, with a high degree of concordance with tissue biopsy, they can identify both clonal and subclonal mutations, and they can detect resistance substantially earlier than radiographic imaging, which could permit earlier intervention. ${ }^{10,11}$ The first liquid biopsy-based companion diagnostic test was approved by the US Food and Drug Administration in 2016, for the detection of EGFR mutations associated with NSCLC.

Yet, even liquid biopsy alone is not able to fully dissect the extent of tumor heterogeneity, especially because it is limited in its ability to assess spatial heterogeneity. Truly effective assessment of tumor heterogeneity is likely to require a combination of liquid biopsy, carefully selected tumor tissue biopsies, imaging diagnostics, and biomarkers.

The ongoing TRACERx (Tracking cancer evolution through therapy $[\mathrm{Rx}]$ ) trials are evaluating a combination of approaches to follow tumor evolution across the course of treatment. The study in NSCLC began in 2014 with a target enrollment of 842 patients and will follow patients over 6 years. Preliminary data from the first 100 patients were recently published and demonstrated that increased intratumor heterogeneity correlated with increased risk of recurrence or death. ${ }^{12}$

If patients consent, the TRACERx trials also feed into the PEACE (Posthumous evaluation of advanced cancer 
environment) trials, which are collecting postmortem biopsies to further evaluate tumor heterogeneity and evolution. TRACERx trials in several other cancer types are now also underway.

\section{Cutting off the source}

The main therapeutic strategies for overcoming tumor heterogeneity are focused on the mechanisms of resistance that it drives. It is becoming increasingly apparent that rationally designed combinations of drugs are likely to be required and might need to be administered early in the course of disease to prevent resistance.

However, according to mathematical modeling studies, combinations of at least 3 drugs may be necessary. ${ }^{13}$ In many cases, this is unlikely to be feasible owing to the unavailability of drugs for certain targets and issues of toxicity, as well as the high cost.

An alternative strategy is to use immunotherapy, because a single treatment can target multiple neoantigens simultaneously. Although immunotherapy has proved to be a highly effective treatment paradigm in multiple tumor types, resistance still arises through varied mechanisms with tumor heterogeneity at their core. ${ }^{14,15}$

A promising avenue for drug development is to cut off the source of tumor heterogeneity - genomic instability and the mutagenic processes that foster it (Table 2). This is exemplified by the success of poly(ADP-ribose) polymerase (PARP) inhibitors in patients with breast cancer susceptibility (BRCA1/2) gene mutations.

Both germline and somatic mutations in the $B R C A 1 / 2$ genes are observed in $10 \%$ to $15 \%$ of patients with ovarian cancer and a substantial number of patients with other types of cancer, including breast, pancreatic, and prostate cancers. ${ }^{16,17}$

These genes play a central role in the homologous recombination (HR) pathway of DNA repair, which repairs double-strand breaks in DNA. PARP inhibitors target a different DNA repair pathway, base excision repair, which repairs single-strand breaks. The use of PARP inhibitors in patients with $B R C A 1 / 2$ mutations is designed to create irreparable damage to the DNA repair processes and drive an unsustainable level of genome instability that leads to cell death, whereas normal cells without HR deficiency can survive. ${ }^{18}$

A growing number of PARP inhibitors are now approved for use in the United States for the treatment of ovarian cancer. In January, olaparib became the first PARP inhibitor approved for patients with $B R C A 1 / 2-$ mutant breast cancer, based on data from the OlympiAD trial in which 302 patients were randomized to receive olaparib $300 \mathrm{mg}$ twice daily or physician's choice of chemotherapy. Olaparib improved progression-free survival from 4.2 months to 7.0 months (hazard ratio, $0.58 ; P=.0009$ ), and the most common adverse events included anemia, nausea, fatigue, and vomiting. ${ }^{19}$

Tumors with other defects in HR have also shown susceptibility to PARP inhibition, shifting interest toward identifying and treating these tumors as a group, independent of histology - about a quarter of all tumors display HR deficiency. ${ }^{20}$ This novel strategy of targeting mutational processes across a range of tumor types has also been exploited in the development of immunotherapies. 
Patients with defects in the mismatch repair (MMR) pathway and microsatellite instability (MSI) - multiple alterations in the length of microsatellite markers within the DNA - are more sensitive to immunotherapy, likely because they are predisposed to a high level of somatic mutations that can serve as neoantigens to provoke a strong anti-tumor immune response.

In 2017, 2 immune checkpoint inhibitors were approved for use in patients with MSI-high or defective MMR (dMMR) cancers. The indication for pembrolizumab
(Keytruda) was independent of tumor histology, the first approval of its kind. It was based on the results of 5 clinical trials in which 149 patients with MSI-H or dMMR cancers were given pembrolizumab $200 \mathrm{mg}$ every 3 weeks or $10 \mathrm{mg} / \mathrm{kg}$ every 2 weeks for a maximum of 24 months. The overall response rate was $39.6 \%$, including 11 complete responses and 48 partial responses. ${ }^{21}$

\section{A new paradigm}

Treatment of a tumor is one of the major selective pressures

TABLE 3 Select ongoing clinical trials incorporating intratumor heterogeneity

\section{Trial name/ \\ identifier}

Phase

Sponsor

\section{Description}

Adaptive (on and off scheduling) abiraterone therapy for metastatic CRPC; patients will be enrolled who achieve $\geq 50 \%$ decline in their PSA levels while on abiraterone and treatment will not be reinitiated until there is $a \geq 50 \%$ increase in PSA

Intermittent ADT for stage IV castration-sensitive prostate cancer; PSA and testosterone level will be used to guide treatment

$\mathrm{H}$ Lee Moffitt Cance Center and Research Institute

$\begin{array}{llll}\text { NCT03416153 } & 2 & \begin{array}{l}\text { University of Michigan } \\ \text { Cancer Center }\end{array} & \begin{array}{l}\text { Individualized adaptive de-escalated radiotherapy for HPV-related oropharyngeal } \\ \text { cancer; uses pre- and midtreatment imaging to guide de-escalation }\end{array} \\ \text { NCT03122522 } & 2 & \begin{array}{l}\text { Memorial Sloan Kettering } \\ \text { Cancer Center }\end{array} & \text { Adaptive dosing of ipilimumab and nivolumab combination immunotherapy }\end{array}$

\begin{tabular}{|c|c|c|c|}
\hline NCT02771314 & 2 & $\begin{array}{l}\text { Hellenic Oncology } \\
\text { Research Group }\end{array}$ & $\begin{array}{l}\text { Liquid biopsy used as a tool to evaluate resistance to first and third generation EGFR } \\
\text { TKls in EGFR-mutant NSCLC; genetic evolution and biological characteristics of CTCs } \\
\text { will be monitored over time after treatment }\end{array}$ \\
\hline $\begin{array}{l}\text { TRACERx } \\
\text { NCTO1888601 }\end{array}$ & NA & $\begin{array}{l}\text { University College } \\
\text { London }\end{array}$ & $\begin{array}{l}\text { Tracking non-small-cell cancer evolution through therapy; after tumors from diagnosis } \\
\text { to relapse and tracking genetic evolution }\end{array}$ \\
\hline $\begin{array}{l}\text { TRACERx-TNBC } \\
\text { NCT03077776 }\end{array}$ & NA & UNICANCER & $\begin{array}{l}\text { Tracking triple-negative breast cancer evolution through therapy; examining the } \\
\text { relationship between intratumor heterogeneity and response to neoadjuvant } \\
\text { chemotherapy }\end{array}$ \\
\hline NCT02993536 & NA & $\begin{array}{l}\text { Abramson Cancer Center } \\
\text { of the University of } \\
\text { Pennsylvania }\end{array}$ & After the clonal evolution of B cells in high-risk CLL after idelalisib-rituximab treatment \\
\hline NCT0305964 1 & NA & GenePlus-Beijing Co Ltd & $\begin{array}{l}\text { Therapeutic resistance and clonal evolution assessed with liquid biopsy of NSCLC } \\
\text { patients in China }\end{array}$ \\
\hline
\end{tabular}

$\begin{array}{llll}\begin{array}{l}\text { CHRONOS } \\ \text { NCT03227926 }\end{array} & 2 & \begin{array}{l}\text { Fondazione del Piemonte } \\ \text { per l'Oncologia }\end{array} & \begin{array}{l}\text { Evaluating the safety and efficacy of rechallenge with panitumumab driven by Ras } \\ \text { resistance dynamics in patients with metastatic CRC; using liquid biopsy to determine } \\ \text { extended-Ras alterations }\end{array} \\ \text { NCT0342529 } & 1 \quad \begin{array}{l}\text { John Wayne Cancer } \\ \text { Institute }\end{array} & \begin{array}{l}\text { A longitudinal assessment of tumor evolution in patients with brain cancer follow- } \\ \text { ing treatment with temozolomide + RT, ipilimumab monotherapy or ipilimumab + } \\ \text { nivolumab combination therapy }\end{array}\end{array}$

DARWIN I

NCTO2 183883

Deciphering afatinib response and resistance with intratumor heterogeneity; patients registered in the TRACERx study will receive afatinib

\begin{tabular}{|c|c|c|c|}
\hline $\begin{array}{l}\text { DARWIN II } \\
\text { NCT02314481 }\end{array}$ & 2 & $\begin{array}{l}\text { University College } \\
\text { London }\end{array}$ & $\begin{array}{l}\text { Deciphering antitumor response and resistance with intratumor heterogeneity; evalu- } \\
\text { ating the impact of intratumor heterogeneity on anti-PD-L1 therapy }\end{array}$ \\
\hline
\end{tabular}

ADT, androgen deprivation therapy; CLL, chronic lymphocytic leukemia; CTC, circulating tumor cells; EGFR, epidermal growth factor receptor; HPV, human papillomavirus; NSCLC, non-small cell lung cancer; RT, radiation therapy; PD-LI, programmed cell death ligand 1; PSA, prostate specific antigen 
that shapes its evolution and recent evidence has emerged that these selective pressures can be highly dynamic. Study findings have shown that there is a cost associated with evolution of resistant subclones and, if the selective pressure of therapy is removed, that cost may become too high, such that resistant subclones are then outcompeted by drug-sensitive ones. There have been reports of reversal of drug resistance when drug treatment is interrupted.

The current treatment paradigm is to try to eliminate tumors by hitting them hard and fast with the maximum tolerated dose (MTD) of a drug. However, there is increasing appreciation that this may be inadvertently fostering more rapid disease progression because it selects for the emergence of resistant cells and eliminates all their competitors (Figure 2).

This is driving a potential paradigm shift, in which researchers are applying concepts from evolutionary biology and the control of invasive species to the treatment of

\section{References}

1. Dagogo-Jack I, Shaw AT. Tumour heterogeneity and resistance to cancer therapies. Nat Rev Clin Oncol. 2018;15(2):81-94.

2. Dzobo K, Senthebane DA, Thomford NE, Rowe A, Dandara C, Parker MI. Not everyone fits the mold: intratumor and intertumor heterogeneity and innovative cancer drug design and development. OMICS. 2018;22(1):17-34.

3. McGranahan N, Swanton C. Clonal heterogeneity and tumor evolution: past, present, and the future. Cell. 2017;168(4):613-628.

4. Davis A, Gao R, Navin N. Tumor evolution: linear, branching, neutral or punctuated? Biochim Biophys Acta. 2017;1867(2):151-161.

5. Amirouchene-Angelozzi N, Swanton C, Bardelli A. Tumor evolution as a therapeutic target. Cancer Discov. Published online first July 20, 2017. Accessed May 23, 2018. doi: 10.1158/2159-8290.CD-17-0343

6. Wu D, Wang DC, Cheng Y, et al. Roles of tumor heterogeneity in the development of drug resistance: a call for precision therapy. Semin Cancer Biol. 2017;42:13-19.

7. Ferguson LR, Chen H, Collins AR, et al. Genomic instability in human cancer: molecular insights and opportunities for therapeutic attack and prevention through diet and nutrition. Semin Cancer Biol. 2015;35(suppl):S5-S24.

8. Forbes SA, Beare D, Gunasekaran P, et al. COSMIC: exploring the world's knowledge of somatic mutations in human cancer. Nucleic Acids Res. 2015;43(Database issue):D805-811.

9. Rosenthal R, McGranahan N, Herrero J, Swanton C. Deciphering genetic intratumor heterogeneity and its impact on cancer evolution. Ann Rev Cancer Biol. 2017;1(1):223-240.

10. Esposito A, Criscitiello C, Locatelli M, Milano M, Curigliano G. Liquid biopsies for solid tumors: understanding tumor heterogeneity and real time monitoring of early resistance to targeted therapies. Pharmacol Ther. 2016;157:120-124.

11. Venesio T, Siravegna G, Bardelli A, Sapino A. Liquid biopsies for monitoring temporal genomic heterogeneity in breast and colon cancers. Pathobiology. 2018;85(1-2):146-154.

12. Jamal-Hanjani M, Wilson GA, McGranahan N, et al. Tracking the evolution of non-small-cell lung cancer. New Engl J Med. cancer. Instead of completely eliminating a cancer, a strategy of adaptive therapy could be used to set up competition between different subclones and keep tumor growth in check by exploiting the high cost of resistance. ${ }^{22}$

Adaptive therapy involves the use of treatment holidays, intermittent dosing schedules or reduced drug doses, rather than using the MTD. Adaptive therapy was tested recently in mice with triple-negative and estrogen receptor-positive breast cancer. The standard maximum dose of chemotherapy was compared with adaptive therapy with either reduced doses or skipped doses as the tumor responded. Tumor growth initially decreased with all 3 treatment scenarios, but then regrew when chemotherapy was stopped or doses were skipped. However, adaptive therapy with lower doses resulted in long-term stabilization of the tumor where treatment was eventually able to be withdrawn. ${ }^{23}$ Clinical trials of several different types of adaptive therapy strategies are ongoing (Table 3).

2017;376(22):2109-2121

13. Bozic I, Reiter JG, Allen B, et al. Evolutionary dynamics of cancer in response to targeted combination therapy. Elife. 2013;2:e00747.

14. Zugazagoitia J, Guedes C, Ponce S, Ferrer I, Molina-Pinelo S, Paz-Ares L. Current challenges in cancer treatment. Clin Ther. 2016;38(7):1551-1566.

15. Ventola CL. Cancer immunotherapy, Part 3: challenges and future trends. PT. 2017;42(8):514-521.

16. Cavanagh H, Rogers KMA. The role of BRCA1 and BRCA2 mutations in prostate, pancreatic and stomach cancers. Hered Cancer Clin Pract. 2015;13:16.

17. Moschetta M, George A, Kaye SB, Banerjee S. BRCA somatic mutations and epigenetic BRCA modifications in serous ovarian cancer. Ann Oncol. 2016;27(8):1449-1455.

18. Brown JS, O'Carrigan B, Jackson SP, Yap TA. Targeting DNA repair in cancer: beyond PARP inhibitors. Cancer Discov. 2017;7(1):20-37.

19. Robson M, Im S-A, Senkus E, et al. Olaparib for Metastatic Breast Cancer in Patients with a Germline BRCA Mutation. New England Journal of Medicine. 2017;377(6):523-533.

20. Williers H, Pfaffle HN, Zou L. Targeting homologous recombination repair in cancer: molecular targets and clinical applications. In: Kelley M, Fishel M, eds. DNA repair in cancer therapy. 2 nd ed: Academic Press; 2016:119-160.

21. U.S. Food and Drug Administration. FDA grants accelerated approval to pembrolizumab for first tissue/site agnostic indication. 2017; https:/www.fda.gov/Drugs/InformationOnDrugs/ ApprovedDrugs/ucm560040.htm. Accessed May 1st,, 2018.

22. Gallaher JA, Enriquez-Navas PM, Luddy KA, Gatenby RA, Anderson ARA. Adaptive Therapy For Heterogeneous Cancer: Exploiting Space And Trade-Offs In Drug Scheduling. bioRxiv. 2017.

23. Enriquez-Navas PM, Kam Y, Das T, et al. Exploiting evolutionary principles to prolong tumor control in preclinical models of breast cancer. Sci Transl Med. 2016;8(327):327ra324. 